\title{
Conserved methionine 165 of matrix protein contributes to the nuclear import and is essential for influenza $A$ virus replication
}

\author{
Petra Švančarová and Tatiana Betáková (1)
}

\begin{abstract}
Background: The influenza matrix protein (M1) layer under the viral membrane plays multiple roles in virus assembly and infection. $\mathrm{N}$-domain and $\mathrm{C}$-domain are connected by a loop region, which consists of conserved RQMV motif.

Methods: The function of the highly conserve RQMV motif in the influenza virus life cycle was investigated by sitedirected mutagenesis and by rescuing mutant viruses by reverse genetics. Co-localization of $\mathrm{M} 1$ with nucleoprotein $(\mathrm{NP})$, clustered mitochondria homolog protein (CLUH), chromosome region maintenance 1 protein (CRM1), or plasma membrane were studied by confocal microscopy.

Results: Mutant viruses containing an alanine substitution of R163, Q164 and V166 result in the production of the virus indistinguishable from the wild type phenotype. Single M165A substitution was lethal for rescuing infection virus and had a striking effect on the distribution of M1 and NP proteins. We have observed statistically significant reduction in distribution of both M165A ( $p<0,05)$ and NP $(p<0,001)$ proteins to the nucleus in the cells transfected with the reverse-genetic system with mutated M1. M165A protein was co-localized with CLUH protein in the cytoplasm and around the nucleus but transport of M165-CLUH complex through the nuclear membrane was restricted.

Conclusions: Our finding suggest that methionine 165 is essential for virus replication and RQMV motif is involved in the nuclear import of viral proteins.
\end{abstract}

Keywords: Influenza a virus, M1 protein, NP protein, CLUH, Reverse genetic

\section{Background}

Influenza A virus is a major respiratory pathogen. The genome contains eight segments of negative sense, single stranded RNA, each encapsidated into ribonucleoproteins by the viral RNA-dependent RNA polymerase and multiple copies of the viral nucleoprotein. The approximately $13 \mathrm{~kb}$ genome encodes up to 16 proteins [1-4].

The matrix protein $\mathrm{M} 1$ is a multifunctional protein playing many essential roles throughout the virus life cycle. M1 protein is a major structural protein, which associates with ribonucleoprotein and the cytoplasmic tails of haemagglutinin and neuraminidase by forming a

\footnotetext{
* Correspondence: virupebo@savba.sk; virubeta@savba.sk

Biomedical Research Center - Slovaks Academy of Sciences, Institute of Virology, Bratislava, Slovak Republic
}

matrix layer underneath the lipid bilayer of the viral envelope $[5,6]$. This protein plays key roles in structural integrity, replication, virus assembly and budding $[7,8]$.

The $M 1$ protein is encoded by the $M$ gene segment and comprised of 252 amino acids. This $\alpha$-helical protein consists of two domains: N-terminal domain from 1 to 165 aa and C-terminal domain from 165 to 252 aa, which are linked by a protease sensitive loop. Although the crystallisation attempts on the full-length of M1 protein, only the structures of $\mathrm{N}$-domain could be determined as the $\mathrm{C}$-terminal region was digested during crystallisation and therefore missed the final crystal $[5,9]$. The N-terminal fragment has a globular structure and consists of nine $\alpha$-helices and eight loop regions, and does not include any $\beta$-strands. According to analysis using programs for 
secondary structure prediction, the C-domain consists of three $\alpha$-helical regions. $\mathrm{N}$-domain and $\mathrm{C}$-domain are connected by a loop region, which forms small $\alpha$-helix (aa 162-166). The conservative MQMV motif presented in the $\alpha$-helix possesses a glutamine-methionine cleavage site [10].

The multiple functional motifs (aa 91-105) includes nuclear localization signal (NLS) (RKLKR) involved in translocation of M1 from the cytoplasm into the nucleus and in binding of vRNA and vRNP [11, 12]. The zinc finger motif (aa 148-162) has been shown to associate with zinc molecules to inhibit viral replication, and is important for interaction with RNP [13-15]. The arginine triplet R76/77/78 participate in M1 interaction with membranes [16]. The oligomerization pattern of M1 is controlled by residues 181-193 and forms highly conserved region in all subtypes of influenza A virus [17]. N-terminal domain of M1 mediates protein-protein contact with NP [11, 18]. Baudin et al. [19] found that C-terminal domain bounds to vRNA and NP alone whilst Noton et al. [20] concluded that for the binding of NP is primarily responsible the middle domain of M1. Interaction of M1 with vRNPs is crucial for nuclear export of vRNPs-M1-NEP complex via CRM1-dependent pathway [7, 19, 21-25].

In the present study, we have investigated the role of the putative glutamine-methionine cleavage site in the RQMV motif in replication. The RQMV motif is conserved in all influenza A strains. We have used the site-direct mutagenesis and rescue of mutant viruses by reverse genetic systems to define the function of this region in the virus life cycle. Crucially, we showed that alanine substitution of methionine 165 was lethal for virus replication and affected the distribution of viral and cellular proteins.

\section{Materials and methods Cells and viruses}

293 T (ATCC CRL-3216) and MDCK (ATCC CCL-34) cells were grown in Eagle's Minimum Essential Medium (EMEM) containing $10 \%$ calf serum in a $5 \% \mathrm{CO}_{2}$ atmosphere at $37^{\circ} \mathrm{C}$. The rescued viruses were growth on MDCK cells. Viral titers were determined by plaque assay.

\section{Antibodies}

Mouse monoclonal antibody raised against anti-influenza A, Puerto Rico 8/34 (H1N1), Bangkok 1/79 (H3N2), matrix protein (I7650-19C) was purchased from US Biological. The rabbit polyclonal anti-CRM1 antibodies (ab24189), anti-NP antibodies (ab104870), and anti-sodium potassium ATPase antibody(ab197713) plasma membrane marker (AlexaFluor488) were obtained from Abcam, rabbit polyclonal anti-CLU1/ KIAA0664 antibody (LS-C288546) was ordered from LS BIO. Rabbit antisera were produced to the peptide corresponding to the C-terminal sequence SAVDVDDGHFVNIELE of the M2 protein conjugated to keyhole limpet haemocyanin [26]. Alexa Fluor 555 goat anti-mouse $\operatorname{IgG}(\mathrm{H}+\mathrm{L})$ (A11008) and Alexa Fluor 488 donkey anti-rabbit IgG $(\mathrm{H}+\mathrm{L})$ (A31572) (Invitrogen) were used as secondary antibodies for immunofluorescence microscopy.

\section{Plasmid construction}

Viruses with mutated M1 protein were generated using the plasmid-based reverse genetics system, a kindly provided by Dr. Y. Kawaoka [27]. The eight plasmids contain the cDNA of the virus A/WSN/33 (H1N1). Plasmids pHW187-M encoding the mutant M1 proteins R163A, Q164A, M165A, and V166A were prepared using Phusion Site-Directed Mutagenesis Kit (Finnzymes). Sequences of oligonucleotide primers and details of cloning are available upon request. All constructs were sequenced to confirm that they lacked unwanted mutations. We also confirmed the functionality of the resulting plasmids by reverse genetics. DNA was purified using Pure Yield ${ }^{\mathrm{Tm}}$ Plasmid Maxiprep (Promega).

\section{Generation of viruses by reverse genetics}

Plasmid-based reverse genetics for virus generation were performed as previously described [25]. Briefly, the eight plasmid containing the cDNA of the virus poll -polII system for generation of A/WSN/33 (H1N1) virus were transfected into co-cultured $293 \mathrm{~T}$ and MDCK cells using TurboFect Transfection reagent (Thermo Scientific). Plasmids pHW187-M encoding the mutant M1 proteins R163A, Q164A, M165A, and V166A were used to rescue of mutant viruses. At $48 \mathrm{~h}$ posttransfection, culture supernatants were harvested and inoculated into MDCK cells for virus propagation. After $48 \mathrm{~h}$, cell culture media were centrifuged to remove cell debris, and the supernatants were stored as stock viruses. The titers of the stock viruses were determined by plaque assays in MDCK cells as was previously published [28]. All viruses were sequenced to confirm the presence of wanted mutations. Prepared A/WSN/33 virus was used as control wild type virus (VC).

\section{Western blotting}

The cell sediment was lysed in extraction buffer $(1 \%$ Triton-X-100, 1mMEDTA, 20mMTris-HCl, pH 7.4) containing proteinase inhibitor Sigma Fast Protease Inhibitors (Sigma). After $10 \mathrm{~min}$ on ice, the lysates were clarified by microcentrifugation for $1 \mathrm{~min}$, and the supernatants were analysed by electrophoresis on $12.5 \%$ polyacrylamide gels. Immunoblotting was done as described by Betakova et al. [29] using rabbit anti-M2, Protein A-horseradish peroxidase conjugate (BioRad) and 3,3'-Diaminobenzidine (DAB) Enhanced Liquid Substrate System tetrahydrochloride (Sigma). 


\section{Immunofluorescence assay}

Co-cultured $293 \mathrm{~T}$ and MDCK cells were grown on glass coverslips and transfected with eight RNA polymerase I plasmids as described above. After $24 \mathrm{~h}$, the cells were fixed with $3 \%$ paraformaldehyde or methanol, permeabilised with $0.01 \%$ Triton in phosphate-buffered saline (PBS) and immunolabelled with primary antibodies $(1 \mu \mathrm{g} / \mathrm{ml})$ diluted in PBS containing $1 \%$ bovine serum albumin (BSA). Primary antibodies were visualised using fluorescein or rhodamine conjugated secondary antibodies diluted in PBS, 1\% BSA. The nuclei were labelled for 10 min with DAPI.

\section{Microscope image acquisition}

Confocal laser scanning was performed using a Zeiss LSM 510 Meta fitted with a Plan Apochromatic $\times$ 40/1.4 oil objective lens. The Z-stack images were taken in a range from 0.1 to $0.5 \mu \mathrm{m}$. Images were collected at 16 bits and at a resolution of 1024 by 1024 pixels. The image processing and analysis were carried out using Fiji/ImageJ software.

\section{Statistical analyses}

Significant differences in the expression of proteins between the control group (M1) and M165A were calculated using the unpaired Student's t-test. $P$ values $<0.05$ were considered to be significant. Statistical analysis was performed using Graph-Pad Prism software.

\section{Results}

\section{M165 is essential for virus replication}

To test the role of single amino acids in the RQMV motif, we have generated plasmids with single mutation in M1 gene. The recombinant viruses were rescued by transfection in co-cultured $293 \mathrm{~T}$ and MDCK cells. Since anti-M1 antibody did not work in western blot, we used anti-M2 polyclonal serum to compare expression of M2 protein after transfection with M1 (control virus - VC),
R163A, Q164A, M165A, and V166A plasmids (Fig. 1a). Mutation in M1 protein did not affect expression of M2 protein. Although M2 protein could not be used for M1 protein measurement, it was used to compare transfection efficiency. The expression of M1 was checked by indirect fluorescent microscopy (data not shown). The expression of M1 and M2 proteins from all plasmids was comparable. All viruses were rescued except M165A mutant, when the M2 protein was not detected in MDCK cells re-infected with medium from transfected cells (Fig. 1b). The lack of M165A virus replication in re-infected MDCK cells was confirmed by RT-PCR. The viral RNA was detected only in the MDCK cells re-infected with supernatant from VC, R163A, Q164A, and V166A mutants (Fig. 1c).

\section{Replication of rescued mutant viruses is comparable with control virus}

Generated single point M1 mutants were further characterized. The growth kinetics of these viruses was determined using MDCK cells. There were no obvious differences among control wild type virus (VC) prepared by reverse genetic system and R163A, Q164A and V166A mutant viruses in the growth curves (Fig. 2). The appearance of revertants was not observed after 6 passages in MDCK cells.

\section{M165A blocked transport of NP into the nucleus}

To understand the role of M165 in virus replication, we examined the effect of M165A mutation on the transport of M1 and NP in transfected cells. Eight RNA polymerase I plasmids (reverse genetic) were transfected into co-cultured $293 \mathrm{~T}$ and MDCK cells. At $24 \mathrm{~h}$ posttransfection, the cells were fixed and labelled with anti-M1 and anti-NP as described in material and methods. M1 and M165A were observed in the cytoplasm and nucleus. There were differences in the proportion of $\mathrm{M} 1$ and M165A mutated protein in the nuclei of transfected cells

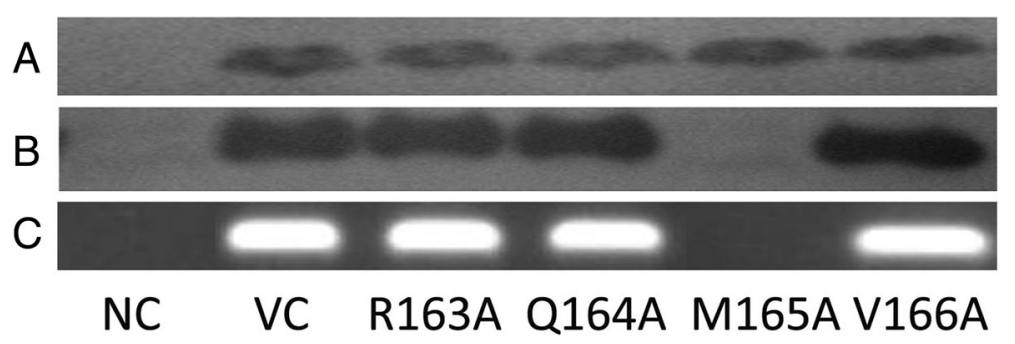

Fig. 1 Confirmation of effective transfection in co-cultured 293 T and MDCK cells and virus production in MDCK cells. The expression of viral proteins was detected in transfected co-cultured 293 T and MDCK cells (a) and in re-infected MDCK cells (b) at $48 \mathrm{~h}$ post transfection/infection by western blotting with anti-M2 polyclonal antibodies. (c) Detection of mRNA M1 in infected MDCK cells. The MDCK cells were infected with supernatant from co-cultured $293 \mathrm{~T}$ and MDCK cells. At $48 \mathrm{~h}$ post-infection, the cell lysates were used for RNA purification and mRNA specific to M1 was determined by RT-PCR 


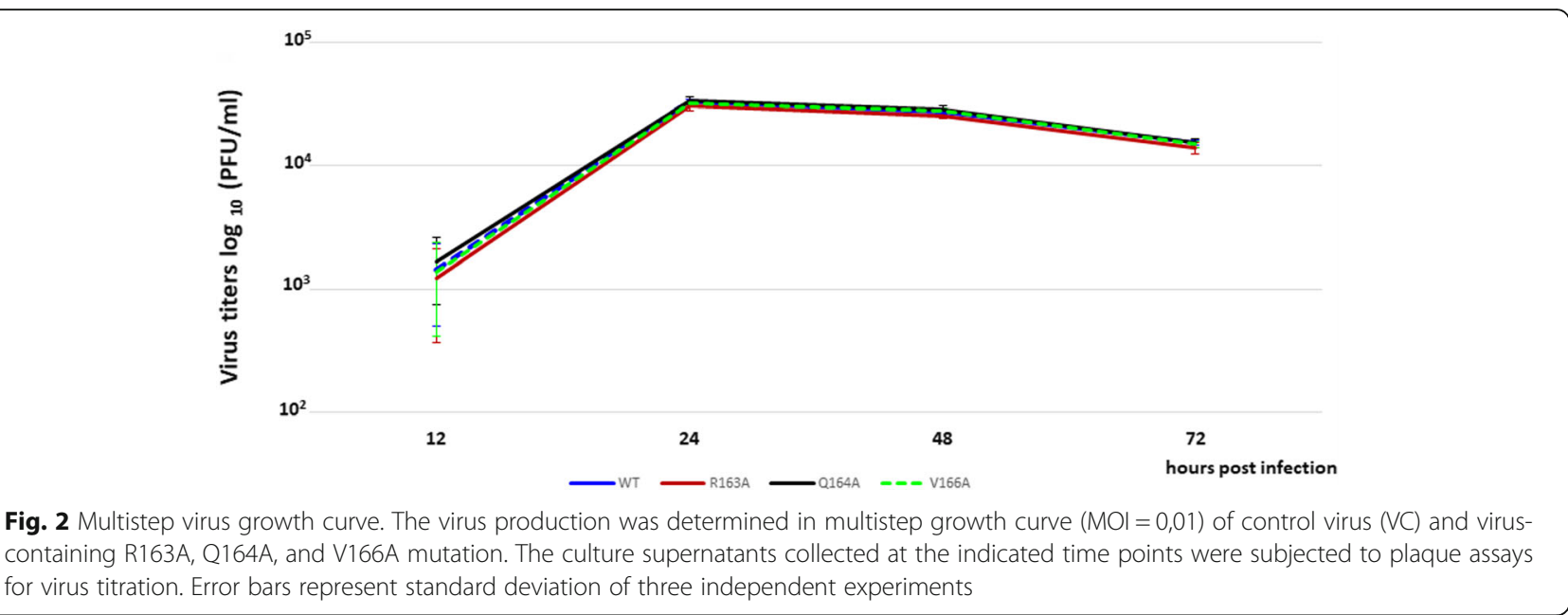

(Fig. 3a). The pictures obtained by confocal fluorescent microscopy were analysed using Fiji/Image J software and the total signals/pixel in the nucleus was calculated. Compare to M1, the amount of M165A protein was significantly reduced in the nuclei $(p<0.05)$ (Fig. 3b).

Since M1 mediates the binding to NP, we were interested to determine differences in the binding of these two proteins. Subcellular localization of NP protein was examined in the cells transfected with both M1 and M165A proteins. As expected, the predominant nuclear localization of NP was found in the cells transfected with M1 (Fig. 3a). On the other hand, NP expressed in the cells transfected with M165A was co-localized with M165A protein in the cytoplasm and around the nucleus. The quantification analyses showed that the nuclear signal of NP protein in the cells transfected with M1 is significantly lower $(p<0.01)$ in comparison with NP in the cells transfected with M165A (Fig. 3b). Although the co-localization of NP and both M1 and M165A proteins were not disrupted, the migration pattern of M165 and especially NP in the cells transfected with M165A was completely different. Mutation M165A resulted in blockage of NP and M1 transport into the nucleus.
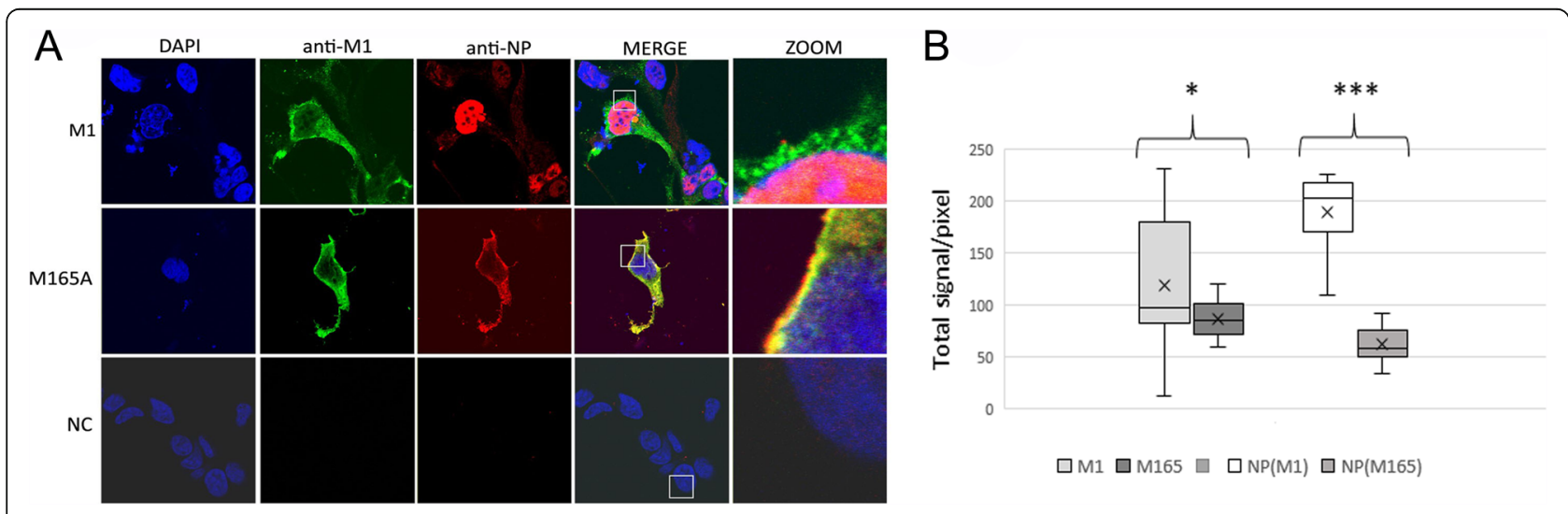

Fig. 3 Cellular co-localization and quantification of NP with M1 and M165A mutant using immunofluorescence confocal microscopy. a/ Co-cultured 293 T and MDCK cells were transfected with eight plasmids for reverse genetic system. After $24 \mathrm{~h}$, the cells were fixed and M1 and M165A were detected using a primary anti-M1 antibody and a secondary antibody coupled to Alexa488 (in green) and NP protein was labelled with anti- NP antibody and a secondary antibody coupled with Alexa555 (in red). Nuclei were stained with DAPI (in blue). NC represents non transfected cells. The intracellular distribution of M1 and NP was imaged by confocal laser scanning fluorescence microscopy (LSM Zeiss 510 Meta). Insets show higher magnification views of the selected areas. The results are representative of three independent experiments. $\mathbf{b} /$ The images obtained from fluorescent microscopy were quantified using Fiji/lmageJ software. The data were delivered from the measurement of 30 cells. The graph indicated mean \pm SD. Statistical analysis was performed using t-test. A $p$-value $<0.05$ was considered statistically significant. NP(M1) is NP in the cells transfected with M1, NP(M165) is NP in the cells transfected with M165A mutant 
M165A influences CLUH translocation to the nucleoplasm To determine co-localization M165A and CLUH, cocultured $293 \mathrm{~T}$ and MDCK cells were transfected with eight plasmids for reverse genetic system where M1 was replaced by $\mathrm{M} 165 \mathrm{~A}$ mutant and CLUH localization was analysed. CLUH plays a role in the subnuclear transport of progeny vRNP to the assembly site of viral nuclear export complexes via nuclear speckles. Normally, CLUH localizes in the cytoplasm. And in influenza virus-infected cells, CLUH translocases from the cytoplasm to the nucleus [30]. In the cells transfected with M1, CLUH was imported into the nucleus (Fig. 4). In M165A transfected cells, transport into the nucleus was impaired and CLUH was co-localized with M165 in the cytoplasm and around the nucleus.

\section{Association of M165A protein with CRM1 around the nucleus}

CRM1 has been identified as an export factor for the leucine-rich nuclear export signal (NES) [32]. In untrasfected cells, CRM1 was localized in the nucleus and nuclear membrane was strongly labeled (Fig. 5). Transfection with M1 and M165A resulted in increased amount of the CRM1 protein in the nucleus. Unlike M1 protein co-localized with CRM1 protein in the nucleus, M165A protein was co-localized with CRM1 protein on the cytoplasmic face of the nuclear membrane.
M165A is associated with the plasma membrane

M165A protein was broadly co-localized with plasma membrane. M1 does not normally traffic to the plasma membrane when expressed alone unless it is artificially recruited to the plasma membrane by addition of a targeting peptide or is overexpressed using a vaccinia virus expression system $[32,33]$. M1 can be recruited to the plasma membrane by HA or NA via the respective cytoplasmic tail [34, 35], and also by the M2 protein [32]. To determine whether M165A mutation change the M1 presence at the plasma membrane, the co-cultured $293 \mathrm{~T}$ and MDCK cells were transfected as described above and plasma membrane were labelled with the anti-sodium potassium ATPase antibody (Fig. 6). Mutation M165A did not interrupt M1 association with the host cellular membrane.

\section{Discussion}

In this study, we have analysed the RQMV motif which is highly conserved among all avian and mammals' viruses. Alanine substitution for R163, Q164, and V166 in $\mathrm{M} 1$ protein did not affect viral growth and distribution of M1 protein inside the cells. Mutation V166A is presented in some avian viruses and thereby it is not surprising that this mutation is well tolerated in our system. Only mutation of M165 to alanine resulted in the failure of virus production. Reverse genetic experiments were repeated more than 20 times and several incubation periods, such as 24, 48, 72 and $96 \mathrm{~h}$ were tested.

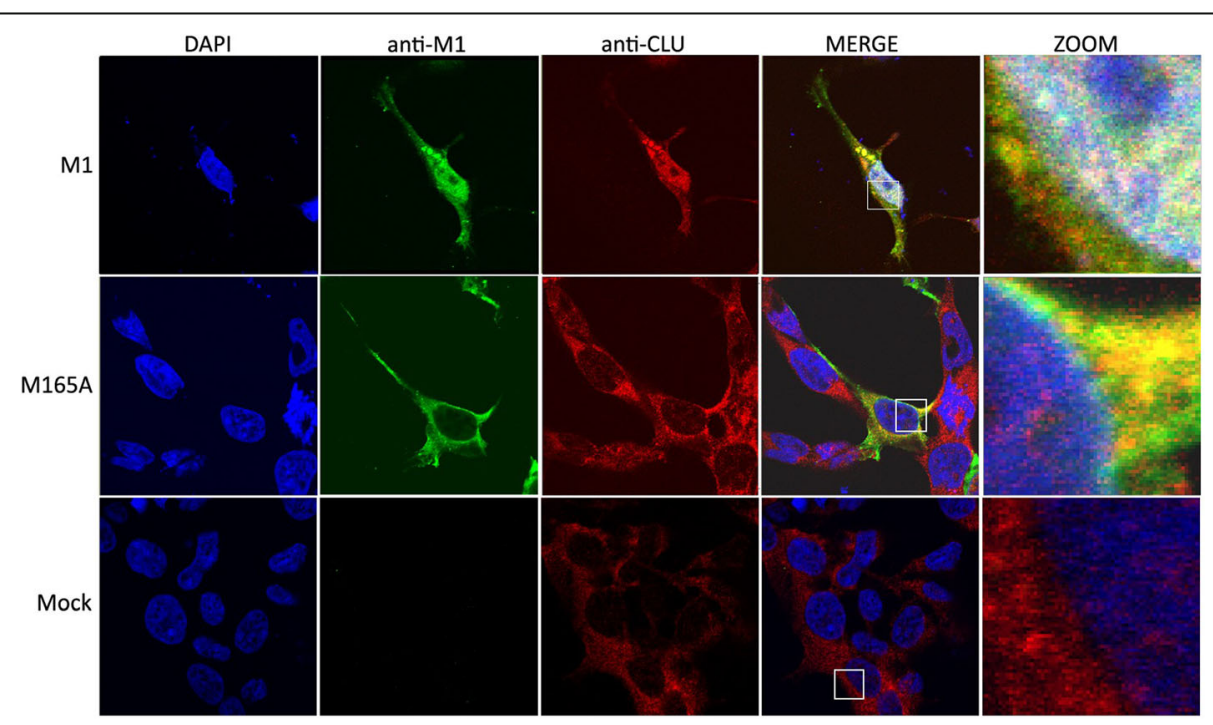

Fig. 4 Cellular co-localization of CLUH with M1 and M165A mutant using immunofluorescence confocal microscopy. Co-cultured 293 T and MDCK cells were transfected with eight plasmids for reverse genetic system. After $24 \mathrm{~h}$, the cells were fixed and M1 and M165A were detected using a primary anti-M1 antibody and a secondary antibody coupled to Alexa488 (in green). CLU protein was labelled with anti- CLUH antibody and a secondary antibody coupled with Alexa555 (in red). Nuclei were stained with DAPI (in blue). NC represents non transfected cells. The intracellular distributions of M1 and CLUH were imaged by confocal laser scanning fluorescence microscopy (LSM Zeiss 510 Meta). Insets show higher magnification views of the selected areas. The results are representative of three independent experiments 


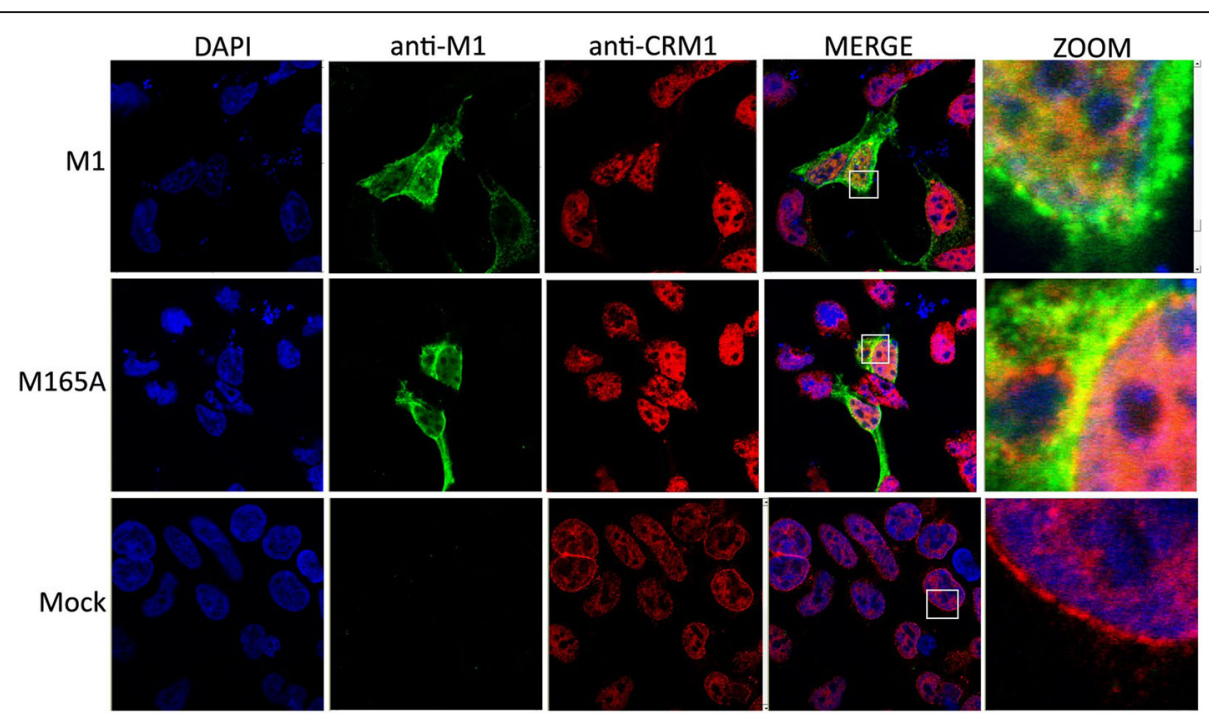

Fig. 5 Cellular co-localization of CRM1 with M1 and M165A mutant using immunofluorescence confocal microscopy. Co-cultured 293 T and MDCK cells were transfected with eight plasmids for reverse genetic system. After $24 \mathrm{~h}$, the cells were fixed and M1 and M165A were detected using a primary anti-M1 antibody and a secondary antibody coupled to Alexa488 (in green). CRM1 protein was labelled with anti-CRM1 antibody and a secondary antibody coupled with Alexa555 (in red). Nuclei were stained with DAPI (in blue). NC represents non transfected cells. The intracellular distributions of M1 and CRM1 were imaged by confocal laser scanning fluorescence microscopy (LSM Zeiss 510 Meta). Insets show higher magnification views of the selected areas. The results are representative of three independent experiments

The plasmids were also used in higher concentrations. We have also prolonged the incubation time after re-infection in MDCK cells but M165A mutant virus was never rescued. We have also tried to make a virus with M165 V mutation and this mutation was also lethal.
Mutation M165A in M1 protein had a striking effect on the distribution of NP protein. In the cells transfected with M1, NP protein was localized mainly in the nucleus unlike the NP in the cells transfected with M165A mutant where it was localized mainly in the cytoplasm

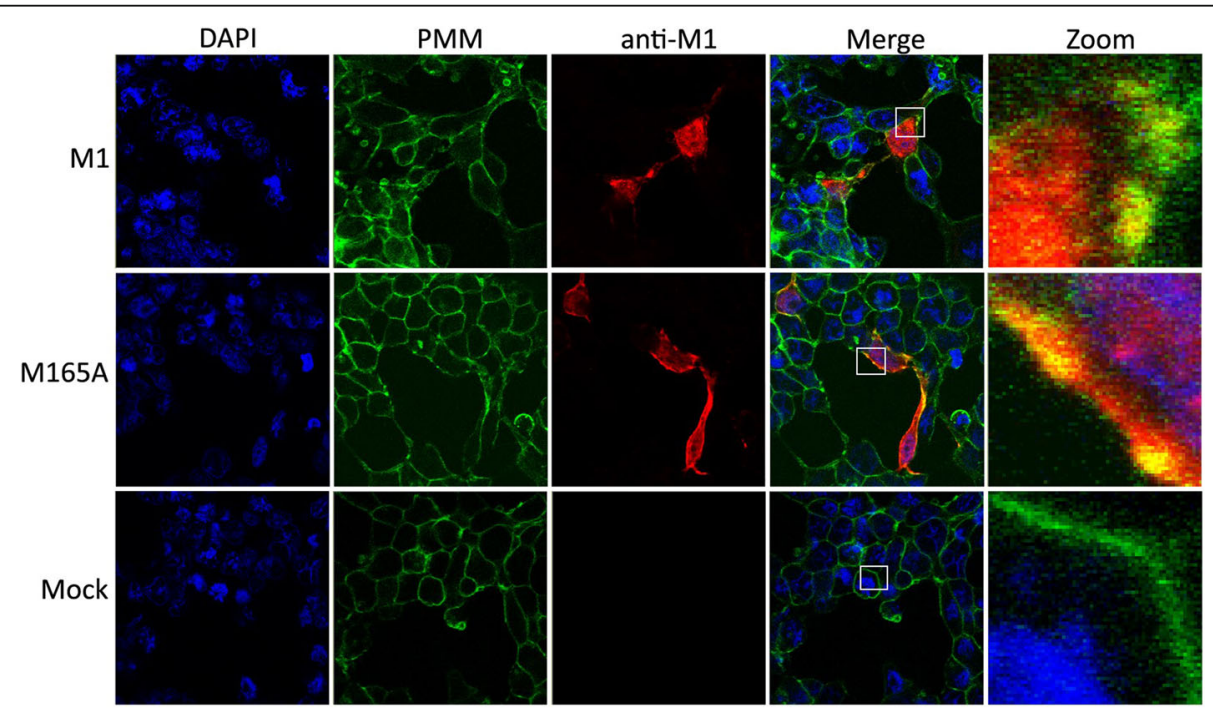

Fig. 6 Cellular co-localization of plasma membrane with M1 and M165A mutant using immunofluorescence confocal microscopy. Co-cultured 293 T and MDCK cells were transfected with eight plasmids for reverse genetic system. After $24 \mathrm{~h}$, the cells were fixed with methanol, plasmatic membrane were labelled with anti-sodium potassium ATPase antibody-plasma membrane marker (Alexa Fluor 488) (in green). M1 and M165A were detected using a primary anti-M1 antibody and a secondary antibody coupled to Alexa555 (in red). Nuclei were stained with DAPI (in blue). NC represents non transfected cells, PMM represents plasma membrane. The plasma membrane and intracellular distributions of M1 were imaged by confocal laser scanning fluorescence microscopy (LSM Zeiss 510 Meta). Insets show higher magnification views of the selected areas. The results are representative of three independent experiments 
and around the nuclear membrane. The amount of NP protein found in the nuclei were analysed by one-way ANOVA and t-test. Differences were considered statistically significant at $\mathrm{p}<0,001$. Surprisingly, differences in localization of M1 protein were less obvious and statistically significant at $p<0,05$.

Transport of M1 protein to the nucleus is mediated by NLS at amino acids 101-105 [36]. It seems that NLS is not the only one responsible for the M1 transport into the nucleus and other protein-protein interaction are suggested. CLUH, a host protein plays a key role in the subnuclear transport of vRNP. Viral PB2 and M1 induce CLUH translocation to the nucleoplasm and SC35-positive speckles. CLUH is usually cytoplasmic and in virus - infected cells is CLUH translocated from the cytoplasm to the nucleus [30]. In M165A transfected cells, M165A-CLUH complexes were detected mostly in the cytoplasm and around the nucleus. The amount of CLUH detected in the nucleus was much lower than that in M1 transfected cells. The physiological functions of CLUH is not fully understood. Translocation of M1 protein depends on association with the CLUH in cytoplasm. Methionine has the propensity to interact with aromatic-containing residues, including tryptophan, tyrosine, and phenylalanine. The Met-aromatic motif is prevalent in many known protein structures and stabilizes protein-protein binding interactions [37]. M165 may stabilize the structure of M1 protein and might affect its interaction with other cellular proteins.

Interaction of M1 and NP proteins is mainly dependent on the positive-negative charge and is described in connection with the vRNP export from the nucleus $[38,39]$. NP is the major protein in the vRNA and plays important role also in vRNP nuclear import [40]. The nuclear transport of NP can be detected in the absence of any other viral proteins in NP transfected cells [41]. Nucleo-cytoplasmic shuttling of NP is mediated through interaction with cellular factors CRM1 and importin- $\alpha$ [42-44]. Interaction of NP with CRM1 is crucial for nuclear export of vRNP-NP-M1-NEP complex $[8,24,25,42]$. We have found that mutation M165A does not change the distribution and co-localization pattern with importin- $\alpha$ (data not shown). M165A which is not transported into the nucleus and stays near the nucleus where associates with CRM1 present in this region. We suggest that M165A mutation disturbed the transport of CLUH-M165A complex into the nucleus and blocked the CRM1 translocation into the nucleus.

We were not able to study co-localization of other virus proteins with CLUH, CRM-1 or importin- $\alpha$ in the cell transfected with revers genetic plasmids including M165A mutant since we did not possess suitable antibodies for multiple labelling and transfection with eight different plasmids did not ensure expression of all proteins in one cell. However, we have observed the same distribution of M1 and M165A proteins in the cells transiently co-transfected with plasmid encoding only M1 protein with or without plasmid encoding NP protein, as well as in the cells transfected with five plasmids: pHW182-PB1, pHW181-PB2, pHW183-PA, pHW185-NP, and $\mathrm{pHW} 127 \mathrm{M}$. M1 protein is not usually transported to the plasma membrane when expressed alone unless it is recruited to the plasma membrane by HA, NA, and M2 proteins [32, 34, 35]. Since co-localization of M165A protein with the plasma membrane is not impaired, we assume that neither is affected the transport and interaction of M165A with HA, NA, and M2 proteins.

Although M165A protein accumulates around the nucleus where is co-localized with CLUH and CRM1 proteins, the transport into the nucleus is restricted. We suggest that this restriction influence also transport of NP to the nucleus. There is very close relationship between NP and C-domain (residue 165-252) of M1 and mutation in this region might affect the interaction between NP-M and formation of nuclear export complex. Mutation in M1 and NP can cause defects in nuclear export $[14,46]$. Transport of NP protein can be restricted by accumulated CLUH-M165A and CRM1M165A complexes on the nuclear membrane and/or some cellular proteins involved in the transport could be dysregulated. Still, there is many studies focusing on nuclear export but nuclear import is not fully explained yet. It was suggested that some still unknown viral and host nuclear factors must be involved in regulation transport of vRNP and viral proteins, and our findings support these suggestions $[31,45,46]$.

\section{Conclusions}

We studied the role of RQMV motif in virus replication. Single R163A, Q164A and V166A substitution resulted in the production of viable viruses. Mutation of M165 was lethal and affected transport of M165A and NP proteins to the nuclei of transfected cells. These data suggest that highly conserved RQMV motif is involved in the nuclear import of viral proteins and other mechanism for the subnuclear transport of viral and host factors must exist. Further analyses should be undertaken to identify the mechanism, and M165 might be a potential target for the development of anti-influenza virus drugs.

\section{Abbreviations}

CLUH: Clustered mitochondria homolog protein; CRM1: Chromosome region maintenance 1; M1: Matrix protein; NES: Nuclear export signal; NLS: Nuclear localisation signal; NP: Nucleoprotein; VC: Control virus A/WSN/33 prepared by reverse genetic; vRNA: (influenza genomic) viral RNA 


\section{Acknowledgments}

We thank Lucia Csaderova, PhD. and Martina Labudova, PhD. for help with confocal microscopy analyses, and DipEng Darina Svetlikova for excellent assistance.

\section{Funding}

This research was supported by the Slovak Research and Development Agency, grant No. APW-0676-12, by the Scientific Grant Agency of the Slovak Republic VEGA No. 2/0014/16.

\section{Availability of data and materials}

The datasets analyzed in this study are available from the corresponding author upon reasonable request.

\section{Authors' contributions}

TB conceived and designed the experiments, performed the experiments, analysed the data, and wrote the paper. PŠ performed experiments. Both authors read and approved the final manuscript.

\section{Ethics approval and consent to participate}

Not applicable.

\section{Consent for publication}

Not applicable.

\section{Competing interests}

The authors declare that they have no competing interests.

\section{Publisher's Note}

Springer Nature remains neutral with regard to jurisdictional claims in published maps and institutional affiliations.

Received: 6 April 2018 Accepted: 13 September 2018 Published online: 03 December 2018

\section{References}

1. Wise HM, Foeglein A, Sun J, Dalton RM, Patel S, Howard W, Anderson EC, Barclay WS, Digard P. A complicated message: identification of a novel PB1related protein translated from influenza a virus segment 2 mRNA. J Virol. 2009:83(16):8021-31.

2. Wise HM, Barbezange C, Jagger BW, Dalton RM, Gog JR, Curran MD, Taubenberger JK, Anderson EC, Digard P. Overlapping signals for translational regulation and packaging of influenza a virus segment 2. Nucleic Acids Res. 2011;39(17):7775-90.

3. Wise HM, Hutchinson EC, Jagger BW, Stuart AD, Kang ZH, Robb $\mathrm{N}_{\text {, }}$ Schwartzman LM, Kash JC, Fodor E, Firth AE, Gog JR, Taubenberger JK, Digard P. Identification of a novel splice variant form of the influenza a virus M2 ion channel with an antigenically distinct ectodomain. PLoS Pathog. 2012;8(11):e1002998.

4. Jagger BW, Wise HM, Kash JC, Walters KA, Wills NM, Xiao YL, Dunfee RL, Schwartzman LM, Ozinsky A, Bell GL, Dalton RM, Lo A, Efstathiou S, Atkins JF, Firth AE, Taubenberger JK, Digard P. An overlapping protein-coding region in influenza a virus segment 3 modulates the host response. Science. 2012:337(6091):199-204.

5. Harris A, Forouhar F, Qiu S, Sha B, Luo M. The crystal structure of the influenza matrix protein $\mathrm{M} 1$ at neutral $\mathrm{pH}$ : M1-M1 protein interfaces can rotate in the oligomeric structures of M1. Virology. 2001;289(1):34-44.

6. Zhang K, Wang Z, Liu X, Yin C, Basit Z, Xia B, Liu W. Dissection of influenza a virus $\mathrm{M} 1$ protein: $\mathrm{pH}$-dependent oligomerization of $\mathrm{N}$-terminal domain and dimerization of C-terminal domain. PLoS One. 2012;7(5):e37786.

7. Neumann G, Hughes MT, Kawaoka Y. Influenza a virus NS2 protein mediates vRNP nuclear export through NES-independent interaction with hCRM1. EMBO J. 2000;19(24):6751-8.

8. Elton D, Simpson-Holley M, Archer K, Medcalf L, Hallam R, McCauley J, Digard P. 2001. Interaction of the influenza virus nucleoprotein with the cellular CRM1-mediated nuclear export pathway. J. Virol. 2001;75(1):408-19.

9. Arzt S, Baudin F, Barge A, Timmins P, Burmeister WP, Ruigrok RW. Combined results from solution studies on intact influenza virus $\mathrm{M} 1$ protein and from a new crystal form of its $\mathrm{N}$-terminal domain show that $\mathrm{M} 1$ is an elongated monomer. Virology. 2001;279(2):439-46.
10. Shishkov AV, Bogachevam EN, Dolgov AA, Chulichkov AL, Knyazev DG, Fedorova NV, Ksenofontov AL, Kordyukova LV, Lukashina EV, Mirsky VM, Baratova LA. The in situ structural characterization of the influenza a virus matrix M1 protein within a virion. Protein Pept Lett. 2009;16(11):1407-13.

11. Elster C, Larsen K, Gagnon J, Ruigrok RW, Baudin F. Influenza virus M1 protein binds to RNA through its nuclear localization signal. J. Gen. Virol. 1997;78(Pt7):1589-96.

12. Rossman JS, Lamb RA. Influenza virus assembly and budding. Virology. 2011; 411(2):229-36.

13. Ye ZP, Baylor NW, Wagner RR. Transcription-inhibition and RNA-binding domains of influenza a virus matrix protein mapped with anti-idiotypic antibodies and synthetic peptides. J Virol. 1989;63(9):3586-94.

14. Ye Z, Liu T, Offringa DP, McInnis J, Levandowski RA. Association of influenza virus matrix protein with ribonucleoproteins. J Virol. 1999:73:7467-73.

15. Liu T, Muller J, Ye Z. Association of influenza virus matrix protein with ribonucleoproteins may control viral growth and morphology. Virology. 2002;304(1):89-96

16. Kerviel A, Dash S, Moncorgé O, Panthu B, Prchal J, Décimo D, Ohlmann T, Lina B, Favard C, Decroly E, Ottmann M, Roingeard P, Muriaux D. Involvement of an arginine triplet in M1 matrix protein interaction with membranes and in $\mathrm{M} 1$ recruitment into virus-like particles of the influenza a(H1N1)pdm09 virus. PLoS One. 2016;11(11):e0165421.

17. Zhang K, Wang Z, Fan GZ, Wang J, Gao S, Li Y, Sun L, Yin CC, Liu WJ. Two polar residues within $\mathrm{C}$-terminal domain of $\mathrm{M} 1$ are critical for the formation of influenza a Virions. Cell Microbiol. 2015;17(11):1583-93.

18. Wakefield L, Brownlee GG. RNA-binding properties of influenza a virus matrix protein M1. Nucleic Acids Res. 1989;17(21):8569-80.

19. Baudin F, Petit I, Weissenhorn W, Ruigrok RW. In vitro dissection of the membrane and RNP binding activities of influenza virus M1 protein. Virology. 2001;281(1):102-8

20. Noton SL, Medcalf E, Fisher D, Mullin AE, Elton D, Digard P. Identification of the domains of the influenza a virus M1 matrix protein required for NP binding, oligomerization and incorporation into virions. J. Gen. Virol. 2007; 88(Pt8):2280-90

21. Ma K, Roy AM, Whittaker GR. Nuclear export of influenza virus ribonucleoproteins: identification of an export intermediate at the nuclear periphery. Virology. 2001;282(2):215-20.

22. Akarsu H, Burmeister WP, Petosa C, Petit I, Müller CW, Ruigrok RW, Baudin F. Crystal structure of the M1 protein-binding domain of the influenza a virus nuclear export protein (NEP/NS2). EMBO J. 2003;22(18):4646-55.

23. Chaimayo C, Hayashi T, Underwood A, Hodges E, Takimoto T. Selective incorporation of vRNP into influenza a virions determined by its specific interaction with M1 protein. Virology. 2017;505:23-32.

24. Elton D, Simpson-Holley M, Archer K, Medcalf L, Hallam R, McCauley J, Digard P. Interaction of the influenza virus nucleoprotein with the cellular CRM1-mediated nuclear export pathway. J Virol. 2001;75(1):408-19.

25. Gao S, Wang S, Cao S, Sun L, Li J, Bi Y, Gao GF, Liu W. Characteristics of nucleocytoplasmic transport of $\mathrm{H} 1 \mathrm{~N} 1$ influenza $\mathrm{A}$ virus nuclear export protein. J Virol. 2014:88(13):7455-63.

26. Betakova T, Hay AJ. Evidence that the CM2 protein of influenza C virus can modify the pH of the exocytic pathway of transfected cells. J Gen Virol. 2007;88(Pt8):2291-6.

27. Hoffmann E, Neumann G, Kawaoka Y, Hobom G, Webster RG. A DNA transfection system for generation of influenza a virus from eight plasmids. Proc. Natl. Acad. Sci. U S A. 2000;97(11):6108-13.

28. Svetlikova D, Kabat P, Ohradanova A, Pastorek J, Betakova T. Influenza a virus replication is inhibited in IFN- $\lambda 2$ and IFN- $\lambda 3$ transfected or stimulated cells. Antivir Res. 2010;88(3):329-33.

29. Betakova T, Ciampor F, Hay AJ. Influence of residue 44 on the activity of the M2 proton channel of influenza a virus. J. Gen. Virol. 2005;86(Pt1):181-4.

30. Ando T, Yamayoshi S, Tomita Y, Watanabe S, Watanabe T, Kawaoka Y. The host protein CLUH participates in the subnuclear transport of influenza virus ribonucleoprotein complexes. Nat Microbiol. 2016;1 (8):16062.

31. Fornerod M, Ohno M, Yoshida M, Mattaj IW. CRM1 is an export receptor for leucine-rich nuclear export signals. Cell. 1997;90(6):1051-60.

32. Wang D, Harmon A, Jin J, Francis DH, Christopher-Hennings J, Nelson E, Montelaro RC, Li F. The lack of an inherent membrane targeting signal is responsible for the failure of the matrix (M1) protein of influenza a virus to bud into virus-like particles. J Virol. 2010;84(9):4673-81. 
33. Gomez-Puertas P, Albo C, Perez-Pastrana E, Vivo A, Portela A. Influenza virus matrix protein is the major driving force in virus budding. J Virol. 2000; 74(24):11538-47.

34. Enami $M$, Enami K. Influenza virus hemagglutinin and neuraminidase glycoproteins stimulate the membrane association of the matrix protein. J Virol. 1996;70(10):6653-7.

35. Ali A, Avalos RT, Ponimaskin E, Nayak DP. Influenza virus assembly: effect of influenza virus glycoproteins on the membrane association of M1 protein. J Virol. 2000;74(18):8709-19.

36. Arzt S, Petit I, Burmeister WP, Ruigrok RW, Baudin F. Structure of a knockout mutant of influenza virus $\mathrm{M} 1$ protein that has altered activities in membrane binding, oligomerisation and binding to NEP (NS2). Virus Res. 2004;99(2):115-9.

37. Valley CC, Cembran A, Perlmutter JD, Lewis AK, Labello NP, Gao J, Sachs JN. The methionine-aromatic motif plays a unique role in stabilizing protein structure. J Biol Chem. 2012;287(42):34979-91.

38. Nayak DP, Hui EK, Barman S. Assembly and budding of influenza virus. Virus Res. 2004;106(2):147-65.

39. Xie H, Lin Z, Mosier PD, Desai UR, Gao Y. The compensatory G88R change is essential in restoring the normal functions of influenza a/WSN/33 virus matrix protein 1 with a disrupted nuclear localization signal. J Virol. 2013; 87(1):345-53.

40. Wu WW, Sun YH. Panté N Nuclear import of influenza A viral ribonucleoprotein complexes is mediated by two nuclear localization sequences on viral nucleoprotein. Virol J. 2007:4:49.

41. Yu M, Liu X, Cao S, Zhao Z, Zhang K, Xie Q, Chen C, Gao S, Bi Y, Sun L, Ye X, Gao GF, Liu W. Identification and characterization of three novel nuclear export signals in the influenza a virus nucleoprotein. J Virol. 2012;86(9): 4970-80.

42. Ye Q, Krug RM, Tao YJ. The mechanism by which influenza a virus nucleoprotein forms oligomers and binds RNA. Nature. 2006; 444 (7122): 1078-82.

43. Li J, Yu M, Zheng W, Liu W. Nucleocytoplasmic shuttling of influenza a virus proteins. Viruses. 2015;7(5):2668-82.

44. Gabriel G, Herwig A, Klenk HD. Interaction of polymerase subunit PB2 and NP with importin alpha1 is a determinant of host range of influenza a virus. PLoS Pathog. 2008:4(2):e11.

45. Li J, Zheng W, Hou L, Chen C, Fan W, Qu H, Jiang J, Liu J, Gao GF, Zhou J, Sun L, Liu W. Differential nucleocytoplasmic shuttling of the nucleoprotein of influenza a viruses and association with host tropism. Cell Microbiol. 2017;19(5):e12692

46. Ninpan K, Suptawiwat O, Boonarkart C, Songprakhon P, Puthavathana P, Auewarakul P. Mutations in matrix protein 1 and nucleoprotein caused human-specific defects in nuclear exportation and viral assembly of an avian influenza H7N1 virus. Virus Res. 2017;238:49-62.

Ready to submit your research? Choose BMC and benefit from:

- fast, convenient online submission

- thorough peer review by experienced researchers in your field

- rapid publication on acceptance

- support for research data, including large and complex data types

- gold Open Access which fosters wider collaboration and increased citations

- maximum visibility for your research: over $100 \mathrm{M}$ website views per year

At $\mathrm{BMC}$, research is always in progress.

Learn more biomedcentral.com/submissions 